\section{PSYCAUSE}

Revue scientifique étudiante de

l'École de psychologie de l'Université Laval
UNvERSTE
LAVAL

Faculté des sciences sociales École de psychologie

\title{
DÉMYSTIFIER LES MÉTHODES QUALITATIVES
}

LES STANDARDS RÉDACTIONNELS DE L'APA POUR LA RÉDACTION D'ARTICLES EMPIRIQUES QUALITATIFS

\author{
Valérie DEMERS ${ }^{1 *}$ \\ 'Laboratoire Psychologie et cultures, Université Laval, Québec, Canada \\ *valerie.demers.3@ulaval.ca
}

\section{Pour citer l'article}

Demers, V. (2020). Démystifier les méthodes qualitatives : Les standards rédactionnels de l'APA pour la rédaction d'articles empiriques qualitatifs. Psycause: Revue scientifique étudiante de l'École de psychologie de l'Université Laval, 10(1), 49-60.

\section{Droits d'auteur}

(C) 2020 Demers. Cet article est distribué en libre accès selon les termes d'une licence Creative Commons Attribution 4.0 International (de type CC-BY 4.0) qui permet l'utilisation du contenu des articles publiés de façon libre, tant que chaque auteur ou autrice du document original à la publication de l'article soit cité(e) et référencé(e) de façon appropriée. 


\title{
DÉMYSTIFIER LES MÉTHODES QUALITATIVES
}

\section{LES STANDARDS RÉDACTIONNELS DE LAPA POUR LA RÉDACTION D'ARTICLES EMPIRIQUES QUALITATIFS}

\author{
Valérie DEMERS ${ }^{1 *}$ \\ ${ }^{1}$ Laboratoire Psychologie et cultures, Université Laval, Québec, Canada \\ *valerie.demers.3@ulaval.ca
}

En recherche, dans le domaine de la psychologie, le courant dominant s'appuie sur l'approche hypothético-déductive, et donc sur la formulation d'hypothèses, auxquelles l'utilisation de tests statistiques permet de répondre (Wertz, 2014). La recherche qualitative semble donc moins fermement implantée dans cette discipline que dans d'autres, comme l'anthropologie (Rennie, Watson, \& Monteiro, 2002) ou la sociologie (Wertz, 2014).

Bien que la publication d'études qualitatives en psychologie ait connue une hausse drastique vers la fin des années 80 et surtout les années 90, plusieurs affirment que ce type de recherche demeure en marge de l'establishment scientifique (Rennie, Watson, \& Monteiro, 2002; Wertz, 2014). Cependant, les méthodes qualitatives subissent présentement un processus de textbookification (Polkinhorne, 2005). En effet, les manières de mener des recherches et les critères permettant de les évaluer sont en train de se consolider, de se cristalliser. Puisque ce processus est en cours, les méthodes qualitatives ne semblent pas être encore totalement institutionnalisées (Flick, 2007). Ainsi, la majorité des psychologues n'utilisent pas les méthodes qualitatives pour conduire leurs recherches et, d'ailleurs, les connaissent peu (Wertz, 2014). Ces méthodes restent donc, à ce jour, relativement peu intégrées aux programmes d'études universitaires en psychologie (Wertz, 2014).

II en résulte que les étudiantes et les étudiants ayant réalisé une recherche impliquant des méthodes qualitatives peuvent rester perplexes lorsque vient le temps de rédiger un article présentant leurs résultats, à plus forte raison s'ils désirent le publier dans un périodique scientifique avec comité de lecture. Les différences, notamment méthodologiques, existant entre les méthodes qualitatives et les méthodes quantitatives sont telles que les standards de rédaction s'appliquant à ces dernières ne sont pas toujours pertinents en «quali» (p. ex., mention de l'hypothèse, présentation des tests statistiques). Quels sont donc les éléments cruciaux à inclure dans un article empirique qualitatif? Quels sont ceux qu'il faut omettre? Comment présenter notamment ses analyses, les précautions prises pour garantir la rigueur ainsi que la qualité de ses recherches et ses résultats tout en satisfaisant aux exigences du périodique visé entre autres en termes de longueur maximale à respecter?

La sixième édition du Publication Manual de l'American Psychological Association (APA; 2010), référence clé quant aux standards de rédaction en psychologie ${ }^{1}$, est muette sur l'existence même des méthodes qualitatives... Heureusement, I'APA a mandaté un comité d'expertes et d'experts pour formuler des standards guidant la rédaction d'articles empiriques qualitatifs en psychologie. Ces standards, publiés en 2018 dans la revue American Psychologist (Levitt et coll., 2018), sont intégrés à la septième édition du Publication Manual (Kazak, 2018). Ils sont également présentés sur le site web consacré au style de rédaction

1 Communément appelé «manuel de I'APA». 
de l'APA (http://www.apastyle.org). La publication de ces standards marque un moment historique (Levitt et coll., 2018)! L'APA reconnaît officiellement -et sans ambiguïté- la contribution des méthodes qualitatives aux connaissances scientifiques en psychologie. Le présent article vise à vous présenter ces nouveaux standards rédactionnels ainsi que quelques suggestions pertinentes à la rédaction d'articles empiriques qualitatifs. Voilà qui, je l'espère, vous donnera quelques outils vous permettant de rédiger de tels articles de manière assurée. Auparavant, je définirai ${ }^{2}$ brièvement ce que sont les méthodes qualitatives.

\section{Que sont les méthodes qualitatives?}

Par comparaison avec les méthodes quantitatives, on peut définir grossièrement les méthodes qualitatives comme regroupant les outils scientifiques permettant de recueillir et de traiter des données non numériques, c'est-à-dire verbales ou graphiques (p. ex., couleurs, lignes, espace). Les méthodes qualitatives diffèrent généralement des méthodes quantitatives quant aux fondements épistémologiques (i.e., paradigme) sur lesquels les chercheuses et chercheurs se basent, aux buts et au type de démarche des études, à la sélection des personnes qui y participent, au déroulement des études et aux critères permettant d'évaluer leur qualité. Les tenants des méthodes qualitatives se rallient généralement au constructivisme et refusent l'idée d'une réalité unique pouvant être décrite de manière "neutre» ou "objective» (p. ex., Guba \& Lincoln, 1990). Les études qualitatives mettent de l'avant diverses méthodes visant à explorer et à comprendre la multiplicité des expériences humaines subjectives, uniques et changeantes (p. ex., Guba \& Lincoln, 1990). Contrairement aux méthodes quantitatives, utilisées pour vérifier une hypothèse quantifiable, les méthodes de recherche qualitatives sont idéales pour répondre à des questions de recherche associées à l'exploration, au défrichement d'un domaine de recherche (Miles \& Huberman, 1994; Morrow, 2007), à la description ou à l'approfondissement des connaissances sur un phénomène ou au développement d'une compréhension des expériences subjectives des individus y prenant part (Conseil de recherches en sciences humaines du Canada, Conseil de recherches en sciences naturelles et en génie du Canada \& Instituts de recherche en santé; CRSH, CRSNG \& IRSC, 2014; Polkinghorne, 2005). Les études qualitatives peuvent aussi viser, de par leurs retombées, à critiquer des institutions, à améliorer des services ou à permettre aux participantes et participants (provenant notamment de groupes marginalisés ou sous-représentés) de devenir des co-chercheurs ayant «voix à la recherche» (Rubin \& Rubin, 2005). Certaines études qualitatives ont aussi pour objectif de favoriser l'émergence d'un changement social (CRSH, CRSNG \& IRSC, 2014; voir p. ex., la thèse de Fortin-Pellerin, 2010).

L'intérêt des méthodes qualitatives ne réside pas dans la généralisation des conclusions d'une étude réalisée avec un échantillon à un nombre plus vaste de personnes. Contrairement aux études quantitatives, dont c'est habituellement la visée, les études qualitatives comprennent généralement moins de participantes et de participants. L'intérêt des études qualitatives consiste plutôt à comprendre en profondeur un phénomène humain (Polkinhorne, 2005), qu'il soit vécu par une seule personne, plusieurs personnes disséminées ou encore un groupe. Les échantillons qualitatifs regroupent un nombre variable de participantes et de participants selon le type de méthode mis de l'avant et les buts de l'étude (CRSH, CRSNG \& IRSC, 2014). Les échantillons qualitatifs sont cependant habituellement plus restreints que leurs cousins quantitatifs. Les individus participant aux études qualitatives sont souvent choisis via l' «échantillonnage dirigé » (Patton, 1990); il s'agit de sélectionner les personnes les plus susceptibles de contribuer à informer sur le phénomène d'intérêt. Les données sont fréquemment analysées non pas après, mais plutôt pendant la collecte de données. À un certain moment, les nouvelles personnes prenant part à l'étude cessent d'apporter de nouvelles informations et se mettent à répéter ce que les précédentes ont déjà apporté. On dit alors que les données ont atteint le point de saturation empirique (voir p. ex., Pirès, 1997). La saturation empirique, originant des travaux de Glaser et Strauss (1967) sur la théorie ancrée, est largement acceptée comme critère

2 Le lectorat intéressé peut consulter la première partie de cet article, titré Les différences entre le « quali » et le « quanti », paru dans la revue Psycause, volume 9, numéro 1, p. 72-82. Note: La section «Que sont les méthodes qualitatives? " se base notamment sur le module numérique enrichi utilisé dans le cadre du cours de premier cycle Méthodes de recherche (PSY-1005), rédigé en collaboration avec Tamarha Pierce, Ph. D., professeure titulaire à l'École de psychologie de l'Université Laval. 
indiquant ${ }^{3}$ que le recrutement peut être interrompu, puisque l'ensemble des facettes du phénomène ciblé a été couvert (Saunders et coll., 2018). Recruter d'autres individus ne ferait que continuer à dédoubler l'information déjà analysée.

En raison des différences entre méthodes qualitatives et quantitatives, apprécier leur qualité peut difficilement se faire à l'aide des mêmes critères de scientificité. Plusieurs ensembles de critères distincts permettent donc d'évaluer la qualité des études qualitatives. Un ensemble de critères, dits "parallèles», ont été formulés par Lincoln et Guba (1985; Guba \& Lincoln, 1990) de manière à transposer en quelque sorte les critères de qualité quantitatifs usuels à la recherche qualitative. Ces critères peuvent être compris comme jouant donc un rôle similaire à la crédibilité, la transférabilité, la fiabilité et la confirmabilité ${ }^{4}$. D'autres critères sont utilisés lors d'études dont les retombées visent à favoriser un changement social ou politique. C'est le cas des critères dits "relationnels» (p. ex., équité ou fairness) et des authenticités ontologique, éducative, catalytique et tactique (Lincoln, 1995; Lincoln \& Guba, 2000).

Aborder plus en détail les critères et les choix méthodologiques permettant de garantir la qualité des études qualitatives est impossible, compte tenu des visées du présent article. C'est d'autant plus le cas que certaines traditions de recherche (p. ex., théorie ancrée) se basent sur des procédures et critères de qualité qui leur sont propres. Rappelons que les méthodes qualitatives, mobilisent de nombreux critères de qualité pour assurer leur scientificité.

\section{Les standards rédactionnels de l'APA : des repères pour rédiger en qualitatif}

Maintenant que nous avons survolé quelques aspects spécifiques aux méthodes qualitatives, abordons les outils pouvant faciliter la rédaction d'un article empirique qualitatif.
Précisons que les standards rédactionnels que propose I'APA (Levitt et coll., 2018) ne sont en aucun cas exhaustifs! L'identification des éléments à inclure dans un article, qualitatif ou quantitatif, exigera toujours réflexion et esprit critique. C'est d'autant plus le cas qu'il existe une grande variabilité dans les études qualitatives, notamment sur le plan des méthodes ( $p$. ex., par observation participante, par groupe focalisé, par entrevue) et du paradigme (bases épistémologiques) qui les sous-tend ( $p$. ex., constructivisme, théorie critique). Ne vous laissez pas impressionner par les spécificités des méthodes qualitatives, qui peuvent paraitre complexifier la rédaction d'un article empirique! Les standards de rédaction proposés par l'APA (Levitt et coll., 2018) offrent des repères flexibles permettent de cerner ce qu'il convient d'y inclure ou non. Levitt et ses collègues insistent d'ailleurs sur l'importance du jugement des autrices ou auteurs, qu'ils invitent à adapter de façon souple ces standards aux particularités de leur étude. Voici donc un tour d'horizon de ces repères, basé sur l'article de Levitt et ses collègues $(2018)^{5}$, présentés selon les sections habituelles d'un article empirique: résumé, introduction, méthode, résultats et discussion. Les buts de ces différentes sections seront explicités et accompagnés de quelques conseils fondés sur ma propre expérience en recherche qualitative.

\section{Résumé (abstract)}

Le résumé vise à informer rapidement votre lectorat quant au contenu général de votre article et de son intérêt. Ce résumé doit donc être concis et percutant. Levitt et ses collaborateurs (2018) recommandent d'y inclure les usuels mots-clés permettant aux personnes intéressées de repérer la méthode, le phénomène étudié et les participantes et participants. II est pertinent d'indiquer le paradigme dans lequel s'inscrit votre étude, si cela permet de mieux comprendre son utilité (Levitt et coll., 2018).

3 Le concept de saturation empirique peut être conçu et conceptualisé de différentes manières. J'ai présenté ici la définition de ce concept qui me semble la plus répandue et la plus compréhensible.

4 Pour plus d'information, la lectrice ou le lecteur peut aussi se référer à l'article Démystifier les méthodes qualitatives, partie 1: Les différences entre le "quali» et le "quanti», publié dans le volume 9, numéro 1 de la revue Psycause.

5 Comme il est d'usage de le faire dans un article scientifique, les propos des auteurs, notamment Levitt et coll. (2018), leur ont été attribués explicitement. Les autres propos, pour lesquels je n'ai pas indiqué de références, sont de mon crû. 


\section{Introduction}

Le but de cette section est de présenter la problématique et de justifier la pertinence d'étudier ce phénomène (APA, 2010). Cette section devrait souligner l'originalité des travaux de recherche, comparativement à ce qui a déjà été fait. Comme c'est le cas pour toute étude scientifique, la problématique doit être située dans le contexte plus large des travaux théoriques et empiriques réalisés sur le sujet (Levitt et coll., 2018). II ne s'agit pas seulement de mentionner des travaux contribuant à ce champ d'études, mais bien de présenter une vision globale de ce champ, en introduisant de manière critique les cadres théoriques pertinents, les débats et les controverses ainsi que les lacunes de ces travaux (Levitt et coll., 2018). Cette présentation doit mener à voir la pertinence de l'étude, au-delà de son aspect purement novateur. Bref, le lectorat doit comprendre pourquoi il est important ou intéressant de mener une telle recherche. Ce peut être par exemple parce que votre étude vise à vérifier un cadre théorique donné ou qu'elle vise à mieux connaître un phénomène afin de mieux intervenir auprès des personnes concernées. Le fait que votre étude soit qualitative ne doit pas vous faire écarter des études quantitatives (ou mixtes), car leur omniprésence dans la littérature ferait en sorte que vous négligiez des références essentielles sur le sujet.

L'introduction d'un article quantitatif mène à la formulation d'une question de recherche, puis à une présentation des hypothèses et de ses objectifs spécifiques (APA, 2010). Si, comme la plupart des études qualitatives, la vôtre ne s'appuie pas sur une hypothèse, vous devez plutôt présenter ses objectifs ou les questions de recherche auxquelles elle tente de répondre (Levitt et coll., 2018). Ce peut être d'explorer ou de décrire un phénomène peu connu ou de construire un cadre théorique. II est recommandé de mentionner dans l'introduction le paradigme ou le cadre théorique dans lequel s'inscrit votre étude (p. ex., constructivisme, féminisme), particulièrement s'il s'agit d'un positionnement très novateur (p. ex., recherche coopérative expérientielle; Heron \& Reason, 2000) et si cela éclaire vos objectifs et vos questions de recherche (Levitt et coll., 2008). Vous devez aussi indiquer clairement quelles méthodes ont été employées pour recueillir vos données et quelles méthodes vous permettront de les analyser (Levitt et coll., 2008). Votre lectorat doit aussi pouvoir comprendre en quoi ces méthodes vous permettent d'atteindre vos objectifs et de répondre à vos questions de recherche (Levitt et coll., 2008), bien que ces éléments puissent être développés plus en profondeur dans la section «méthode». Ainsi, tout bien considéré, I'introduction d'un article empirique qualitatif diffère peu de celle d'un article «quanti ».

\section{Méthode}

La section "méthode» vise essentiellement à décrire les choix méthodologiques faits par l'équipe de recherche et à justifier leur pertinence, notamment en lien avec les objectifs ou les questions de recherche (Levitt et coll., 2018). Cela permet entre autres au lectorat d'évaluer la qualité de l'étude, et éventuellement, de la répliquer (APA, 2010, p. 29).

Cette section devrait débuter par un résumé des méthodes de collecte et d'analyse des données et une justification de leur pertinence en regard des objectifs et/ou des questions de recherche, et, si la chercheuse ou le chercheur le juge pertinent, une explication du paradigme sur lequel se base son étude (Levitt et coll., 2018). Cette section comprend habituellement plusieurs autres sous-sections, une structure qui facilite la compréhension du lectorat. Par exemple, une sous-section traite des sources de données (p. ex., individus, documents), une du recrutement, une de la collecte de données et une de l'analyse (Levitt et coll., 2018). Voyons les informations principales que ces sous-sections devraient comprendre.

\section{Participants ou sources de données.}

Si les données sont des documents, la chercheuse ou le chercheur doit les décrire (p. ex., articles de journaux, lettres ou correspondances personnelles), préciser sur combien d'entre eux l'étude s'appuie et expliciter le processus par lequel ils ont été choisis (Levitt et coll., 2018). Si les données ont été recueillies auprès de participantes et participants ${ }^{6}$, la chercheuse ou le chercheur doit les décrire de manière détaillée,

6 Les chercheuses et chercheurs œuvrant en qualitatif se refusent généralement à utiliser les termes «sujets» ou «échantillon », qu'ils jugent trop objectifiants, au profit des termes "participantes et participants», à la connotation reflétant mieux leur collaboration active au processus de recherche. 
permettant ainsi au lectorat d'évaluer la transférabilité (Levitt et coll., 2018), c'est-à-dire l'applicabilité des résultats à d'autres contextes ou à d'autres individus. Cette description doit inclure leurs caractéristiques sociodémographiques et/ou culturelles les plus pertinentes. Par exemple, si une étude qualitative se penche sur les stéréotypes qu'expriment des individus selon leur orientation sexuelle auto-déclarée et leur sexe, le niveau d'éducation et l'intensité des croyances religieuses des participantes et participants pourraient être pertinents à évoquer, puisque ce sont des facteurs pouvant être liés à l'expression de stéréotypes homophobes. En revanche, que les individus prenant part à l'étude soient en couple ou non semble ici bien moins pertinent! Suivant une logique de transparence, les chercheuses ou chercheurs doivent aussi expliciter dans l'article les relations qu'ils avaient avec les participantes ou participants préalablement à leur implication dans l'étude, puisque ces relations sont susceptibles d'influencer les résultats.

\section{Recrutement.}

La section "méthode» doit aussi comprendre une discussion de la procédure employée dans l'étude, c'està-dire des processus de recrutement et de sélection des participantes ou participants (Levitt et coll., 2018).

\section{Processus de recrutement.}

La chercheuse ou le chercheur doit clarifier comment il a recruté les participantes et participants (p. ex., envoi de messages de recrutement à l'aide de listes de distribution, affichage dans les lieux publics, échantillonnage boule de neige). Toute compensation ou incitation financière, de même que l'adhésion de l'équipe de recherche aux règles usuelles d'éthique, doivent aussi être mentionnées (Levitt et coll., 2018).

C'est ici aussi que la chercheuse ou le chercheur explique comment le nombre de participantes ou participants a été établi, et ce, en lien avec les buts ou objectifs de recherche. Si ce nombre a été déterminé par la saturation empirique (Pirès, 1997), il convient de l'évoquer (Levitt et coll., 2018). Comme il a été précisé auparavant, cette saturation permet de s'assurer qu'une bonne partie du phénomène d'intérêt, tel qu'il se manifeste auprès des participantes et participants, a été exploré7; elle contribue donc considérablement à la qualité méthodologique de l'étude. La section «méthode» de l'article est également l'endroit où mentionner l'attrition; si des participantes ou participants ont abandonné en cours d'étude, leur nombre et leurs raisons de désistement doivent être détaillées (Levitt et coll., 2018).

\section{Sélection des participants.}

La chercheuse ou le chercheur doit expliquer la manière dont elle ou il a choisi les participants ou les sources de données, qu'il s'agisse d'un échantillonnage de convenance (p. ex., échantillonnage boule de neige; Levitt et coll., 2018) ou d'un échantillonnage dirigé (purposeful sampling; Patton, 1990). Rappelons-le, l'échantillonnage dirigé, courant dans les études qualitatives, est un processus volontaire par lequel l'équipe de recherche inclut délibérément certaines personnes dans son étude, en raison de leur potentiel à lui permettre de mieux comprendre le phénomène. Ainsi, une des "stratégies» utilisées est de choisir l'échantillon le plus diversifié possible, ce qui permettra de comprendre les multiples formes que peut prendre le phénomène tel que vécu chez ces individus (Pirès, 1997). Présenter les critères d'inclusion et d'exclusion de l'échantillon (Levitt et coll., 2018) est essentiel, de même qu'évoquer le contexte général dans lequel les données ont été recueillies (c'est-à-dire à quel moment et à quel endroit).

\section{Collecte de données.}

Cette sous-section permet de préciser la manière dont ont été recueillies les données (p. ex., observation, questionnaires, entrevues, groupes de discussion). C'est aussi ici que la chercheuse ou le chercheur justifie son protocole de collecte de données et, s'il y a lieu, son évolution dans le temps, dans le cas où les méthodes de collecte de données ont changé en cours de route (Levitt et coll., 2018). Par exemple,

7 Comme il a déjà été précisé, la saturation empirique se reconnaît à la répétition, au dédoublement des mêmes informations parmi les nouveaux individus recrutés. Par exemple, lors d'une analyse de contenu, lors de l'atteinte du seuil de saturation, les personnes nouvellement recrutées n'apportent que des informations pouvant être classée dans les catégories (ou «codes») déjà existantes et ne menant pas à créer de nouvelles catégories. Vous me demanderez: «Combien de ces répétitions doiventelles avoir lieu? » J'aimerais bien vous offrir une réponse claire. II n'existe cependant pas de critère " officiel » indiquant l'atteinte du seuil de saturation. II revient à la personne qui conduit la recherche de déterminer ce seuil et de démontrer son atteinte au lectorat (et au comité de lecture de son article), notamment en fonction de ses objectifs de recherche. 
lors d'une étude par groupes focalisés (focus groups), quelques participantes ou participants ont mentionné à une chercheuse, hors enregistrement, être gênés de s'exprimer publiquement sur le sujet abordé. À la suite de cet événement, cette chercheuse pourrait décider de remplacer les groupes focalisés par des entrevues individuelles, pour éviter de "perdre de données » en raison de la «timidité » des participantes ou participants ${ }^{8}$.

Décrire le ou les processus de collecte de données est aussi un incontournable (Levitt et coll., 2018): y a-t-il eu une ou plusieurs collectes de données? Quelle était sa ou leur durée? Dans quel contexte s'estelle ou se sont-elles effectuée(s)? Qui était présent? Des précisions doivent aussi être données sur les méthodes utilisées pour recueillir les données (Levitt et coll., 2018). Par exemple, dans le cas d'entrevues ou de groupes focalisés, il faut rapporter le type de questions (fermées ou ouvertes) et leur contenu tandis que dans le cas d'observation, il faut spécifier le type dont il s'agit (p. ex., observation systématique, observation participante). Levitt et ses collaborateurs (2018) soulèvent également la nécessité d'aborder les méthodes d'enregistrement (p. ex., audio, vidéo, prise de notes de terrain) et la manière dont la transcription a été réalisée, le cas échéant (p. ex., transcription verbatim, c'est-à-dire pour laquelle le discours des personnes présentes a été écrit intégralement).

Si la collecte des données a été réalisée via observation, la chercheuse ou le chercheur doit aussi discuter de l'«étendue» (extensiveness) de son engagement auprès des participantes ou participants, c'est-à-dire de sa profondeur (p. ex., à quel point elle ou il s'est impliqué(e)), de la fréquence et de la durée de sa présence dans leur milieu (Levitt et coll., 2018). Cette sous-section peut aussi permettre de discuter de I'importance que la chercheuse ou le chercheur a accordé à la réflexivité (Levitt et coll., 2018). Grosso modo, la réflexivité consiste pour la chercheuse ou le chercheur à s'engager dans une démarche de réflexion sur soi-même et sur sa possible influence sur les processus de recherche et les résultats qui en découlent. II s'agit d'un retour sur soi, ses biais, ses a priori, ses raisons de faire ce type de recherche et la manière dont elle ou il a géré ses biais et sa subjectivité (p. ex., en tenant un journal de bord, par des séances de débriefing avec des collègues; Levitt et coll., 2018).

\section{Analyse.}

Écrire cette sous-section méthodologique risque d'être dépaysant pour celles et ceux habitués au «quanti », puisqu'elle traite de ce qui constitue l'étape primordiale ayant permis d'obtenir des données qualitatives: I'analyse. Une telle sous-section risque d'être également plus longue pour une étude qualitative que pour une étude quantitative (Levitt et coll., 2018).

\section{Stratégies d'analyse.}

La chercheuse ou le chercheur doit décrire et justifier son choix de stratégies pour analyser les données, encore et toujours en lien avec les buts de l'étude (Levitt et coll., 2018). Dans une optique de transparence, elle ou il doit aussi détailler les processus sous-tendant l'analyse des données (p. ex., analyse thématique de contenu visant à identifier, organiser, décrire et interpréter les thèmes ressortant d'un texte (Braun \& Clarke, 2006; L'Écuyer, 1990), théorie ancrée, visant à produire une théorie ou un cadre théorique à partir de données qualitatives empiriques; Glaser \& Strauss, 1967). Cette analyse se base-t-elle sur une méthode reconnue? Quelles sont les étapes ayant été suivies? Dans quel ordre ont-elles été effectuées? Par exemple, si les résultats proviennent d'une analyse de contenu, il faut préciser quelles étaient les unités d'analyse ( $p$. ex., thèmes, phrases, paragraphes) et pourquoi. Les catégories (codes) étaient-elles prédéterminées ou les analystes les ont-elles fait émerger des données, par un processus inductif? Si un logiciel d'analyse qualitative (p. ex., NVivo, QDA Miner) a été utilisé, il faut l'indiquer, ainsi que la version dont il s'agit. Si cela n'a pas déjà été fait dans d'autres sections de l'article, cette sous-section est un bon endroit pour préciser qui sont les analystes, codeuses ou codeurs, quelle a été leur implication respective dans l'analyse, ainsi que, s'il y a lieu, la formation qu'ils ont reçue (Levitt et coll., 2018).

\section{Intégrité méthodologique.}

L'expression "intégrité méthodologique» réfère à la qualité (en d'autres termes, à la «scientificité» ou

8 II va sans dire qu'un tel changement dans les méthodes de collecte de données doit être approuvé par le comité éthique de son institution avant d'être mis en place par la chercheuse. 
encore à la rigueur) provenant spécifiquement des choix méthodologiques de l'équipe de recherche. La sous-section traitant de l'intégrité méthodologique regroupe la description des procédures utilisées et qui renforcent cette intégrité. De façon très globale, ce sont des procédures qui assurent que les affirmations de la chercheuse ou du chercheur ne sont pas "gratuites»: les résultats et l'interprétation doivent être justifiés, fidèles et utiles (Levitt et coll., 2018). Par ailleurs, certaines autrices ou auteurs discuteront de ces procédures tout au long de leur article, dans d'autres sections (p. ex., méthode, stratégies d'analyse).

L'intégrité méthodologique implique notamment:

1) Des données adéquates, en ce qu'elles reflètent une certaine diversité du phénomène étudié, pertinente quant aux buts de l'étude et à l'approche adoptée (Levitt et coll., 2018). Par exemple, si j'entreprends d'étudier l'orientation sexuelle et d'en brosser un portrait juste, je devrai probablement rencontrer des personnes non seulement homosexuelles ou hétérosexuelles, mais également pansexuelles ${ }^{9}$, sapiosexuelles ${ }^{10}$, etc.

2) Des résultats bien ancrés dans les données, et non dans l'imagination (potentiellement débordante), de la chercheuse ou du chercheur (Levitt et coll., 2018). Une des manières les plus répandues de le démontrer consiste à présenter les thèmes que l'analyse a permis de faire ressortir des données et à les accompagner d'extraits des données brutes (p. ex., de livres, de journaux, de citations des discours des participantes ou participants). Cela permet au lectorat de vérifier lui-même le lien entre ces données et l'interprétation qui en est faite (Levitt et coll., 2018).

3) Une gestion transparente de la subjectivité. La chercheuse ou le chercheur est responsable d'expliquer commentelle ou il a géré ses perspectives et ses biais, tant durant la collecte de données que durant l'analyse (Levitt et coll., 2018). Par exemple, un chercheur peut tenir un journal de bord, dans lequel il note ses réflexions, ses croyances, ses biais, ses pensées, ses réactions et ses émotions durant toutes les étapes de réalisation de son étude (Cohen \& Crabtree, 2008).
Cette manière de procéder favorise sa réflexivité et sa prise de conscience de son influence sur ses résultats et sur ses interprétations.

4) Une présentation des résultats et des interprétations cohérentes, ne rejetant pas les cas "négatifs» ou contradictoires. Lorsque la chercheuse ou le chercheur formule ses résultats et ses interprétations, elle ou il doit absolument tenir compte de toutes les données (Levitt et coll., 2018) décrivant le phénomène à l'étude, même celles qui «ne font pas son affaire», puisqu'elles contribuent aussi à ce phénomène.

5) Cette section peut aussi permettre de décrire les processus de vérification supplémentaires utilisés, s'il y a lieu, et qui contribuent à accroître la qualité de l'étude (Levitt et coll., 2018). En voici quelques exemples.

a. Demander des rétroactions des participantes et participants sur les résultats et les interprétations que la chercheuse ou le chercheur en fait (member checks; Lincoln \& Guba, 1985) permet de confirmer qu'elle ou il n'est pas «dans le champ». Cependant, cette pratique est critiquée. Elle est longue, complexe et les participantes ou participants désirent souvent clarifier, justifier ou transformer leurs propos précédents, ce qui amène des questionnements (Cohen \& Crabtree, 2008; Poland, 2002): quelles sont les données «authentiques» devant avoir préséance sur les autres? Les informations telles que recueillies au départ? Leur version amendée ou révisée?

b. Consensus entre différents analystes ou codeuses et codeurs. Plusieurs individus analysent indépendamment les données ou une partie d'entre elles. Si leurs points de vue ne sont pas similaires, ils discutent et débattent jusqu'à arriver à s'entendre. La logique qui sous-tend cette procédure est que les résultats sont plus "solides» si plusieurs analystes y sont arrivés que si une seule ou un seul analyste y est arrivé(e) sans que ses interprétations soient remises en question ${ }^{11}$.

9 Une personne pansexuelle est une personne dont le désir ou l'attirance amoureuse s'exerce sans égard à l'orientation sexuelle ou à l'identité de genre de ses "cibles" (pansexual, n.d.).

10 Un individu sapiosexuel est attiré émotivement ou amoureusement par des personnes en fonction de leur intelligence.

11 Bien que ce ne soit pas un élément abordé par Levitt et coll. (2018), I'utilisation d'une mesure d'accord interjuge (p. ex., kappa de Cohen) est utilisée, dans certaines études, pour mesurer le degré auquel divers analystes s'entendent quant à l'identification 
c. Triangulation des analystes ou des sources de données. La triangulation consiste à multiplier le nombre d'analystes ou de codeuses ou codeurs. Le fait que plusieurs personnes arrivent indépendamment aux mêmes résultats ou aux mêmes conclusions à partir des mêmes données réduit de beaucoup la possibilité que ces résultats ou conclusions soient le fruit de leur imagination. La triangulation des sources de données fonctionne de manière similaire: arriver aux mêmes conclusions avec différentes sources de données (p. ex., entrevues individuelles, groupes focalisés et documents écrits) vient «confirmer»la pertinence de ces conclusions.

d. D'autres méthodes contribuent également à la qualité de l'étude: celles qui accroissent la réflexivité de la chercheuse ou du chercheur, comme la rédaction de notes de terrain, de mémos ou d'un journal de bord, de même que les méthodes de "réduction» synthétisant les données, comme les tableaux ou les matrices.

\section{Résultats}

Dans cette section sont décrits les résultats tirés des données (p. ex., les catégories et sous-catégories décrivant le contenu des entrevues ou des documents), ainsi que leur interprétation (Levitt et coll., 2018): qu'est-ce que ces résultats veulent dire? Qu'est-ce le lectorat doit en comprendre? La chercheuse ou le chercheur doit aussi rendre facilement compréhensible le lien entre les données et les résultats présentés: cette section est donc généralement plus longue dans un article qualitatif que quantitatif (Levitt et coll., 2018). De surcroît, le lectorat doit pouvoir apprécier, en lisant des extraits des données brutes, que les interprétations de la chercheuse ou du chercheur ne sont pas exagérées ou même créées de toutes pièces (Levitt et coll., 2018). Pour rendre les résultats plus aisément compréhensibles en un seul coup d'œil, il peut être utile de les présenter sous forme de diagrammes, de modèles ou de tableaux (Levitt et coll., 2018).

\section{Discussion}

La section « discussion » diffère peu selon qu'il s'agisse d'une étude qualitative ou quantitative. La chercheuse ou le chercheur doit ici interpréter les résultats (Levitt et coll., 2018), soit littéralement leur donner un sens, les commenter (interpréter, n.d.). La personne ayant mené l'étude doit donc discuter de leur signification (en lien avec les questions de recherche) et de leurs principaux apports à la compréhension du phénomène étudié (Levitt et coll., 2018). De quelle manière ces résultats s'inscrivent-ils dans la littérature scientifique sur le sujet? Viennent-ils confirmer les résultats d'autres études? Les remettent-ils plutôt en question? La discussion doit être critique, c'est-à-dire que la chercheuse ou le chercheur doit non seulement exposer sa vision des résultats, mais en proposer des explications alternatives, soit d'autres manières de les comprendre (Levitt et coll., 2018). Ce faisant, elle ou il ne discrédite pas «son » interprétation, mais démontre sa capacité d'envisager d'autres scénarios. Ce n'est pas l'endroit pour présenter des résultats non abordés dans la section du même nom! Ce n'est pas non plus l'endroit pour illustrer les résultats par de nouveaux extraits des données brutes.

Cette section doit aussi inclure une analyse des forces et des faiblesses de l'étude (Levitt et coll., 2018), notamment celles qui sont associées à son intégrité méthodologique. Également, la chercheuse ou le chercheur doit commenter la transférabilité des résultats de son étude (Levitt et coll., 2018), soit la possibilité qu'ils s'appliquent dans d'autres milieux ou contextes ou à d'autres personnes que celles qui y ont pris part. Il s'agit de discuter non seulement si, mais aussi à quel point les résultats sont transférables à ces personnes ou à ces contextes différents. Finalement, les implications des résultats doivent être considérées: que signifient-ils pour la recherche menée sur des sujets similaires? Pour l'intervention auprès de personnes similaires aux participantes et aux participants? Que faut-il en tirer sur le plan de la formulation de politiques (p. ex., publiques)?

d'éléments (p. ex., dans l'analyse de contenu, avoir identifié les mêmes sections de texte comme étant des unités pertinentes à analyser) et/ou quant au codage (i.e., à l'attribution d'un «nom de code» ou de "catégorie» aux mêmes unités). En général, plus l'accord est élevé, plus les analystes s'entendent et plus l'analyse est considérée comme "objective». Cette manière de faire témoigne d'une logique facilement compréhensible pour les personnes formées aux méthodes quantitatives, mais est parfois considérée superflue par certaines qui préfèrent utiliser le consensus. Cela dit, l'un n'exclut pas l'autre: il serait possible de calculer une mesure d'accord interjuge entre analystes, qui confronteraient leurs points de vue et les ferait discuter jusqu'à ce qu'ils convergent. 


\section{Quelques conseils}

Comme ce qui précède devrait l'avoir mis en lumière, I'article qualitatif se base sur la même structure de base que l'article quantitatif. Après tout, un article empirique reste un article empirique! Néanmoins, la rédaction d'un article qualitatif comprend son lot de défis, parmi lesquels contextualiser plusieurs aspects de l'étude. Il est aussi crucial de démontrer de manière convaincante l'ancrage explicite des résultats dans les données brutes. En d'autres mots, la chercheuse ou le chercheur doit mettre en lumière comment les résultats ( $p$. ex., les codes ou les thèmes ayant été identifiés lors d'une analyse de contenu) découlent ou proviennent des données brutes (p. ex., des discours verbatim des participantes et participants). Cela assure le lectorat que les résultats ne sont pas des exagérations douteuses de la part des analystes, ou pire encore, des dérives de leur imagination. La transparence quant aux processus utilisés et quant à la probable influence de la chercheuse ou du chercheur sur la collecte, l'analyse et l'interprétation des données, est indispensable. Les explicitations qui résultent de cette volonté de contextualisation et de transparence tendent à rendre les articles qualitatifs plus longs que les articles quantitatifs. II est donc souvent difficile de faire "entrer» un article qualitatif dans les limites de longueur stipulées par certains périodiques scientifiques (et typiquement prévues pour des articles quantitatifs...). Levitt et ses collaborateurs (2018) en conviennent et proposent que les périodiques allouent au moins 10 pages supplémentaires aux articles empiriques qualitatifs. Si votre objectif est de publier votre article dans un périodique ne tenant pas compte de cette suggestion, ne vous découragez pas! Même s'il peut être ardu d'épurer votre "œuvre», il faut faire contre mauvaise fortune, bon cœur et tenter de voir ce difficile processus d'élagage non comme une punition, mais comme une occasion de développer votre concision, une compétence utile et prisée tant en recherche que dans le marché du travail!

Bien que les méthodes qualitatives soient de plus en plus utilisées et diffusées (Levitt et coll., 2018), il peut arriver que le comité de lecture de votre article soit peu familier avec elles. Je vous conseille donc de vous assurer que les justifications de vos choix méthodologiques et analytiques soient non seulement extrêmement claires, mais aussi compréhensibles pour des néophytes en méthodes qualitatives. Concrètement, qu'apportent ces choix à votre étude? $\mathrm{Ne}$ vous bornez pas à dire que vous avez utilisé un journal de bord pour noter vos réflexions, vos insights et la justification de vos choix méthodologiques et analytiques. Expliquez que ce journal permet d'accroître votre réflexivité et de gérer vos biais, mais surtout mentionnez comment, c'est-à-dire parce qu'il vous permet de relire vos notes détaillant vos réactions et vos réactions à chacune des entrevues que vous avez menées, de réfléchir à vos a priori et de les mettre de côté efficacement. Ou encore, expliquez que vous pouvez faire lire ce journal de bord à une ou un collègue ne collaborant pas à votre étude, pour lui faire identifier les biais ou les a priori susceptibles d'influencer vos résultats ou vos conclusions. Par exemple, cette personne «externe» pourrait remarquer que le seul participant que vous avez exclu des analyses en prétendant qu'il ne répond pas aux questions vous avait, dès la première prise de contact, semblé antipathique. Attention cependant de ne pas adopter un ton pédagogique! Ce n'est pas un cours sur le «quali», mais bien un article scientifique. Prenez aussi soin de ne pas être en mode défensif, mais plutôt de mettre en valeur les forces de votre étude.

Avant de conclure, voici quelques derniers conseils. Ne considérez pas la rédaction comme l'ultime étape à réaliser lorsque l'analyse et la collecte de données sont parachevées. Je vous suggère, et ce, dès la cueillette de données, de prendre des notes sur n'importe quel aspect potentiellement pertinent ou utile pour votre article. Vous en savez assez à ce moment sur les stratégies de recrutement que vous avez employées et sur pourquoi vous les avez choisies pour commencer à rédiger la section «méthode». Si vous tombez par hasard sur un article récent parlant d'un sujet connexe, lisez-le et notez-en les éléments les plus pertinents pour votre article (p. ex., résultats, citations percutantes) dans un document consacré à votre «article en cours». II vous vient une idée brillante pour introduire votre sujet, en plein milieu de la nuit, comme toujours? Notez-la dans ce document ${ }^{12}$. Procéder ainsi vous évite de "laisser traîner» toutes

12 Plus réalistement, vous pouvez, durant la nuit, noter votre idée géniale sur un bout de papier laissé à cet effet sur votre table de chevet, puis, le lendemain matin, la transférer dans ce document. 
ces notes plus ou moins disparates sur votre bureau ou de créer des multitudes de documents que vous perdrez probablement... Vous pourrez ensuite ordonner tous ces éléments en les regroupant selon leurcontenu etselon les sections d'un article empirique. Un peu de ménage et de mise en ordre feront donc de ce document le «plan brut» de votre futur article. Vous pourrez alors étoffer progressivement cette ébauche jusqu'à ce qu'elle soit devenue un article complet, enfin digne d'être publié.

Si j'avais un seul conseil à vous donner, ce serait: ne soyez pas trop perfectionnistes au départ! À l'étape du «plan brut», il peut être nuisible de tenter de faire des phrases complètes et bien formulées. Je vous suggère plutôt de noter les informations que vous prévoyez présenter le plus clairement pour vous, sous une forme de liste avec puces (bullets). Ne perdez pas de temps à embellir inutilement la «structure» temporaire de votre article, susceptible de varier encore avant sa complétion. De plus, il est bien plus facile de raffiner une section déjà structurée logiquement que d'écrire du premier coup une magnifique phrase inutile à cet endroit de votre argumentation! Ordonnez. Ré-ordonnez. Ré-ordonnez. Ensuite, vous pourrez peaufiner. Cela dit, si, dans un élan d'inspiration, vous réussissez à écrire "LA» phrase, efficace et bien formulée, ne vous retenez pas! D'ailleurs, on oublie trop souvent qu'écrire un article n'est pas une démarche linéaire. C'est plutôt un processus itératif, qui demande des dizaines de révisions successives pour arriver à un article fini et satisfaisant. N'oubliez pas non plus que les standards rédactionnels qualitatifs de I'APA sont formulés avec souplesse. Ils laissent la chercheuse ou le chercheur, pour rendre son article plus cohérent ou plus concis, regrouper certaines sections de son article empirique (p. ex., la collecte de données et les résultats ou encore les résultats et la conclusion). Tirez donc parti de cela en optimisant la logique et l'efficacité de vos propos.

Terminons par une petite «tranche de vie»: je trouve parfois difficile d'écrire des liens pertinents et fluides entre les paragraphes ou sections de mon article. Ainsi, avant même de tenter de rédiger des phrases complètes, je tente de prévoir les liens logiques qui uniront mes divers paragraphes ou idées, soit sous forme d'éléments précédés de "puces», soit sous formes de phrases plus complètes, si c'est ce qui me vient à l'esprit. Cela peut donner quelque chose comme
"La pile d'articles 1 dit que les recherches sur le sujet sont surtout quantitatives et mesurent l'orientation sexuelle. Par contre, elles ne définissent pas ce concept. Voir article d'Auteur X (2019), qui dit que c'est problématique. Son argument: comment mesurer ce qu'on n'a pas défini? Ensuite, parler de la pile d'articles 2, qui montre qu'il existe diverses façons de définir théoriquement l'orientation sexuelle.» D'ailleurs, je passe beaucoup de temps à établir ces liens logiques, ce qui me permet de clarifier mes idées. Lorsque mon "canevas de base» est assez développé et que je connais précisément les idées que je veux développer, je peux me consacrer à rédiger des phrases complètes et esthétiques.

Voilà. J'espère que cet article aura quelque peu soulagé vos possibles anxiétés rédactionnelles et qu'il vous aura permis de mieux comprendre les particularités d'un article empirique qualitatif. Comme toute forme de production scientifique, la rédaction recourt à des habiletés qui, heureusement, peuvent se développer avec I'habitude. À vos claviers (ou vos crayons) ... et bonne rédaction!

\section{Références}

American Psychological Association. (2010). Publication manual of the American Psychological Association (6e éd.). Washington, DC, États-Unis: Auteur.

Braun, V., \& Clarke, V. (2006). Using thematic analysis in psychology. Qualitative Research in Psychology, 3(2), 77-101.

Brinkmann, S., Jacobsen, M. H., \& Kristiansen, S. (2014). Historical overview of qualitative research in the social sciences. Dans P. Leavy (Éd.), The Oxford Handbook of Qualitative Research (pp.17-42). New York, É-U.: Oxford University Press.

Cohen, D., \& Crabtree, B. (2008). Qualitative research guidelines project. Récupéré sur http://www. qualres.org/HomeLinc-3684.html

Conseil de recherches en sciences humaines, Conseil de recherches en sciences naturelles et en génie et Instituts de recherche en santé du Canada (2014). Énoncé politique des trois Conseils: Éthique de la recherche avec des êtres humains. Récupéré sur: http://www.ger.ethique.gc.ca/fra/policy-politique/ initiatives/tcps2-eptc2/Default/ 
Creswell, J. W. (2013). Qualitative inquiry and research design: Choosing among five approaches ( $3^{e}$ éd.). Los Angeles, CA, États-Unis: SAGE.

Denzin, N. K., \& Lincoln, Y. S. (1998). Introduction: Entering the field of qualitative research. Dans $\mathrm{N}$. K. Denzin \& Y. S. Lincoln (Éds.), The landscape of qualitative research: Theories and issues (pp. 1-34). Thousand Oaks, CA, É-U.: Sage.

Flick, U. (2007). Managing quality in qualitative research. Londres, Angleterre: SAGE.

Fortin-Pellerin, L. (2010). La représentation sociale de l'empowerment de groupes québécois du mouvement des femmes: "C'est quelque chose qui nous a été enlevé et qu'on ré-acquiert" (Thèse de doctorat, Université Laval, Québec, Canada). Récupéré sur www.theses.ulaval.ca/2010/27217/27217.pdf

Glaser, B. G., \& Strauss, A. L. (1967). The discovery of grounded theory: Strategies for qualitative research. Chicago, IL, États-Unis: Aldine.

Guba, E. G., \& Lincoln, Y. S. (1990). Judging the quality of fourth generation evaluation. Dans E. G. Guba \& Y. S. Lincoln (Éds.), Fourth generation evaluation (pp. 184-227). Newbury Park, CA, États-Unis: SAGE.

Heron, J., \& Reason, P. (2000). The practice of co-operative inquiry: Research 'with' rather than 'on' people. Récupéré sur http://www.human-inquiry.com/ciacadem.htm

Interpréter (n.d.). Dans Dictionnaire Larousse en ligne. Récupéré sur https://www.larousse.fr/ dictionnaires/francais/interpr\%C3\%A9ter/43813

Kazak, A. E. (2018). Editorial: Journal article reporting standards. American Psychologist, 73, 1-2. https:// doi.org/10.1037/amp0000263

L'Écuyer, R. (1990). Étapes de l'analyse de contenu: Modèle général. Dans Méthodologie de l'analyse développementale de contenu. Méthode GPS et concept de soi. (pp. 51-123). Québec, Canada: Presses de l'Université du Québec.

Levitt, H. M., Bamberg, M., Creswell, J. W., Frost, D. M., Josselson, R., \& Suárez-Orozco, C. (2018). Journal article reporting standards for qualitative primary, qualitative meta-analytic, and mixed methods research in psychology: The APA Publications and Communications Board task force report. American Psychologist, 73, 26-46. https://doi.org/10.1037/ amp0000151
Lincoln, Y. S. (1995). Emerging criteria for quality in qualitative and interpretive research. Qualitative Inquiry, 1, 275-289. https://doi.org/10.1177/ 107780049500100301

Lincoln, Y. S., \& Guba, N. K. (1985). Naturalistic inquiry. Beverly Hills, CA, États-Unis: SAGE.

Lincoln, Y. S., \& Guba, N. K. (2000). Paradigmatic controversies, contradictions, and emerging confluences. Dans N. K. Denzin, \& Y. S. Lincoln (Éds.), Handbook of Qualitative Research (2e éd., pp. 163-188). Thousand Oaks, CA, États-Unis: SAGE.

Merriam-Webster (n.d.) Words we're watching: What does «sapiosexual» mean? Récupéré sur https:// www.merriam-webster.com/words-at-play/thehearts-wisdom-what-does-sapiosexual-mean

Miles, M. B., \& Huberman, A. M. (1994). Introduction. Dans M. B. Miles \& A. M. Huberman (Éds.), Qualitative data analysis: An expanded sourcebook (2 éd., pp. 1-15). Thousand Oaks, CA, États-Unis: SAGE.

Morrow, S. L. (2007). Qualitative research in counseling psychology: Conceptual foundations. The Counseling Psychologist, 35, 209-235. https://doi. org/10.1177/0011000006286990

Pansexual (n.d.) Dans Merriam-Webster. Récupéré sur https://www.merriam-webster.com/dictionary/pansexual

Patton, M. Q. (1990). Qualitative evaluation and research methods ( $2^{\mathrm{e}}$ éd.). Newbury Park, CA, États-Unis: SAGE.

Pirès, A. P. (1997). Échantillonnage empirique et recherche qualitative: Essai théorique et méthodologique. Dans J. Poupart, J. P. Deslauriers, L. H. Groulx, A. Laperrière, R. Mayer, \& A. P. Pirès (Éds.), La recherche qualitative: Enjeux épistémologiques et méthodologiques (pp. 133-169). Montréal, Canada: Gaétan Morin.

Poland, B. D. (2002). Transcription quality. Dans J.F. Gubrium \& J. A. Holstein (Éds.), Handbook of interview research: context and method (pp. 629-639). Thousand Oaks, CA, É-U: Sage.

Polkinghorne, D. E. (2005). Language and meaning: Data collection in qualitative research. Journal of Counseling Psychology, 52, 137-145. https://doi. org/10.1037/0022-0167.52.2.137 
Rennie, D. L., Watson, K. D., \& Monteiro, A. M. (2002). The rise of qualitative research in psychology. Canadian Psychology, 43(3), 179-189. https://doi. org/10.1037/h0086914

Rubin, H. J., \& Rubin, I. S. (2005). Qualitative interviewing: The art of hearing data ( $2^{\mathrm{e}}$ éd.). Thousand Oaks, CA, États-Unis: SAGE.
Saunders, B., Sim, J., Kingstone, T., Baker, S., Waterfield, J., Bartlam, B., Burroughs, H., \& Jinks, C. (2018). Saturation in qualitative research: Exploring its conceptualization and operationalization. Quality \& Quantity, 52, 1893-1907. https://doi.org/10.1007/ s11135-017-0574-8

Wertz, F. J. (2014). Qualitative inquiry in the history of psychology. Qualitative Psychology, 1(1). 4-16. https://doi.org/10.1037/qup0000007

\section{Pour citer l'article}

Demers, V. (2020). Démystifier les méthodes qualitatives: Les standards rédactionnels de l'APA pour la rédaction d'articles empiriques qualitatifs. Psycause: Revue scientifique étudiante de l'École de psychologie de l'Université Laval, 10(1), 49-60.

\section{Droits d'auteur}

(C) 2020 Demers. Cet article est distribué en libre accès selon les termes d'une licence Creative Commons Attribution 4.0 International (de type CC-BY 4.0) qui permet l'utilisation du contenu des articles publiés de façon libre, tant que chaque auteur ou autrice du document original à la publication de l'article soit cité(e) et référencé(e) de façon appropriée. 